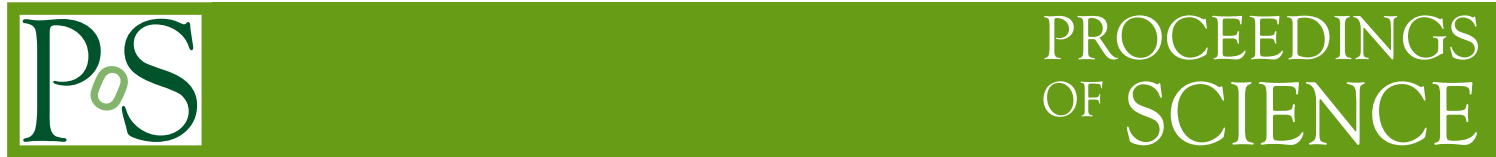

\title{
Chiral Effective Field Theory for EM currents and form factors
}

\section{Stefan Kölling ${ }^{* \dagger}$}

Institut für Theoretische Physik II, Ruhr-Universität Bochum, D-44780 Bochum, Germany

E-mail: stefan.koelling@tp2.ruhr-uni-bochum.de

The application of chiral effective field theory ( $\chi$ EFT) to the nuclear few-body sector has achieved an extremely high level of precision. Calculations of the nuclear potential are performed up to next-to-next-to-next-to leading $\left(\mathrm{N}^{3} \mathrm{LO}\right)$ order in the chiral power-counting. In this contribution, we summarize our efforts to extend the application area of chiral EFT to electromagnetic processes. We present our construction of the electromagnetic current operator consistent with the potential at $\mathrm{N}^{3} \mathrm{LO}$. As an application of our current operator in the calculation of observables, We focus on the electromagnetic form factors of the deuteron, which allow us to determine two low-energy constants.

The 7th International Workshop on Chiral Dynamics, August 6 -10, 2012

Jefferson Lab, Newport News, Virginia, USA

* Speaker.

$\dagger$ I would like to thank the organisers for a very enjoyable workshop and my collaborators Evgeny Epelbaum, Hermann Krebs, Ulf-G. Meißner and Daniel R. Phillips. 


\section{Introduction}

The past two decades saw a very successful application of chiral effective field theory ( $\chi$ EFT) to few-nucleon dynamics at low energies, see Refs. [1,2] for recent reviews. Two-nucleon potentials have been derived to next-to-next-to-next-to-leading order $\left(\mathrm{N}^{3} \mathrm{LO}\right)$ in the chiral expansion $[3,4]$ and they accurately describe existing low-energy scattering data and static properties of the deuteron. Electromagnetic reactions on light nuclei such as elastic electron scattering, photo/electrodisintegration and radiative capture have been extensively studied in nuclear physics. To achieve an accurate description, the knowledge of the electromagnetic current operator, constructed consistently with the nuclear potential, is needed. In their seminal paper [5], Park et. al. were the first to apply $\chi$ EFT to derive the exchange currents. However, this work is limited to threshold kinematics $|\mathbf{q}| \ll M_{\pi}$ with $M_{\pi}$ denoting the pion mass. More recently, this work was extended to the general kinematics suitable to study, e.g., electron scattering off light nuclei at momentum transfer of $|\mathbf{q}|$ of order $M_{\pi}$ by the JLab-Pisa [6, 7, 8, 9] and Bochum-Bonn groups [10, 11, 12].

In this work, we discuss the expansion of irreducible two-nucleon operators $J_{0}$ and $\mathbf{J}$ in powers of $P \equiv\left(p, M_{\pi}\right) / \Lambda$ with $\Lambda$ denoting the hard scale in the theory, e.g. the cutoff $(\sim 600 \mathrm{MeV})$ used in calculations. Following this expansion, the leading-loop order is $e P^{4}$. In calculations of the deuteron form factors one has to keep in mind that most of the corrections to the two-body pieces of the two-nucleon current and charge operators at this order are of isovector type and thus do not contribute. In particular, up to this order, the only two-body contributions to the isoscalar charge density operator, $J_{0}^{(s)}$ emerge from the leading relativistic corrections of one-pion range so that $J_{0}^{(s)}$ is parameter free. The impact of these corrections on the deuteron charge and quadrupole form factors, $G_{C}$ and $G_{Q}$ is studied in Refs. $[13,14]$. In these works the deuteron wave functions obtained from $\chi$ EFT potentials at various orders were used to compute $G_{C}$ and $G_{Q}$ (see also Refs. [15, 16] for earlier work along the same lines). Good agreement with the compilation of elastic electrondeuteron data from Ref. [17] was then found for both form factors in the kinematic range $Q^{2}<0.35$ $\mathrm{GeV}^{2}$, provided factorization was employed in order to account for single-nucleon structure.

The isoscalar two-nucleon current operator $\mathbf{J}^{(s)}$ has two two-body contributions at order $e P^{4}$ : one from a short-distance operator and one of one-pion range. The impact of these terms on the magnetic moments of the deuteron and trinucleons was examined in Ref. [18]. To study the interplay of these terms with each other and with one-body mechanisms, it is illuminating to take a look at the $|\mathbf{q}|^{2}$-dependence of observables. In this contribution we present the results of our study in this direction [12], using $\chi$ EFT expressions for $\mathbf{J}^{(s)}$ derived in Refs. [10, 11] to extend the predictions given for $G_{M}$ in Refs. $[14,16]$ to $O\left(e P^{4}\right)$.

\section{Results}

Since the deuteron is isoscalar, here, we are only interested in the isoscalar part of the exchange currents. This yields a two-body isoscalar current operator $\mathbf{J}^{(s)}[10,11]$ :

$$
\mathbf{J}_{2 B}^{(s)}=2 e \frac{g_{A} i}{F_{\pi}^{2}} \bar{d}_{9} \tau_{1} \cdot \tau_{2} \frac{\sigma_{2} \cdot \mathbf{q}_{2}}{q_{2}^{2}+M_{\pi}^{2}}\left[\mathbf{q}_{\mathbf{1}} \times \mathbf{q}\right]+i e L_{2}\left(\sigma_{1}+\sigma_{2}\right) \times \mathbf{q}_{\mathbf{1}}+(\mathbf{1} \leftrightarrow \mathbf{2}),
$$

where $\mathbf{q}$ labels the photon momentum, $\mathbf{q}_{\mathbf{1} / \mathbf{2}}$ labels the momentum transfer on nucleon one/two respectively and $\bar{d}_{9}$ and $L_{2}$ are low-energy constants (LECs) that parametrize physics not explicitly 
included in $\chi$ EFT. Since $G_{M}$ is determined completely by the one-body part of $\mathbf{J}^{(s)}$ up to $O\left(e P^{3}\right)$ the total form factor is thus

$$
G_{M}=\frac{1}{\sqrt{2 \eta}|e|}\left\langle 1\left|\mathbf{J}_{1 B}^{(s)}+\mathbf{J}_{2 B}^{(s)^{+}}\right| 0\right\rangle
$$

Here we use factorization to compute $\mathbf{J}_{1 B}$, i.e. we write:

$$
\mathbf{J}_{1 B}^{(s)^{+}}=\frac{|e|}{M}\left[G_{E}^{(s)}\left(Q^{2}\right) 2 \mathbf{p}^{+}+i G_{M}^{(s)}\left(Q^{2}\right)\left(\sigma_{1} \times \mathbf{q}\right)^{+}\right]
$$

with $\mathbf{p}$ the momentum of the struck nucleon, and $G_{E}^{(s)}$ and $G_{M}^{(s)}$ the isoscalar single-nucleon form factors, for which we take the parameterization of Ref. [19]. The use of the results from Belushkin et al. for the one-body part of $\mathbf{J}_{1 B}^{(s)^{+}}$is equivalent (up to corrections that begin only two orders beyond the order to which we work) to making a chiral EFT expansion for the "body" form factors $D_{M}$ and $D_{E}$ [20]. Since we are primarily interested in the nuclear effects, we adopt here this more practical approach, which allows us to concentrate on the relevant scales of the two-nucleon system, without having to worry whether the theory describes the isoscalar nuclear structure satisfactory.

We now evaluate the matrix elements in Eq. (2) with a variety of $\chi$ EFT deuteron wave functions computed with the NLO and NNLO $\chi$ EFT potentials and different values of the cutoffs $\Lambda$ in the Lippmann-Schwinger equation and $\tilde{\Lambda}$ in the spectral function. The result found for $G_{M}$ with LO $\chi$ EFT wave functions and the leading piece of $\mathbf{J}^{(s)}$, denoted here as $O(e P)$, was computed in Ref. [21]. Corrections to this come both from higher-order pieces of the NN potential, $V$, which affect the wave function, and from the corrections to $\mathbf{J}^{(s)}$ discussed in the previous section. The NNLO $\chi$ EFT potential includes all effects up to $O\left(P^{3}\right)$ relative to leading (in this counting), so its deuteron wave function, when combined with the $O\left(e P^{4}\right) \mathbf{J}^{(s)}$, yields a $\chi$ EFT calculation for $G_{M}$ which includes all effects up to $O\left(e P^{4}\right)$.

The pertinent matrix elements are computed via Monte-Carlo (MC) integration. To increase efficiency, we use importance sampling with the weight function of Ref. [22]:

$$
p(\mathbf{k}) \equiv p(k)=\frac{(r-3)(r-2)(r-1)}{8 \pi} \frac{C^{r-3}}{(k+C)^{r}} .
$$

The functional form of $p(k)$ is chosen such that the weight function is maximal at the origin, reflecting the large $S$-wave component of the deuteron wave function. The parameters $C$ and $r$ control the vanishing of the weight function at large momenta and are tuned to optimal values (in terms of the efficiency of the MC integration) by calculating the expectation value of the one-pion exchange potential yielding $C=1 \mathrm{GeV}$ and $r=11$.

As in Ref. [22] we perform a path average over several runs. We use 2730 sample points and and the path average is performed for 3000 runs. Analysis of the run-to-run fluctuations indicates a final answer with better than $1 \%$ precision throughout the momentum range of $0-800 \mathrm{MeV}$. At several points we compared this MC answer to calculations using quadrature methods, and always found agreement within the precision claimed.

In order to fix the values of the two LECs entering $\mathbf{J}^{(s)}$, namely $\bar{d}_{9}$ and $L_{2}$ we adopt the following procedure. First, we fix the value of $L_{2}$ for a given $\bar{d}_{9}$ by demanding that the magnetic moment of the deuteron is reproduced. Then, we perform a $\chi^{2}$-fit to the experimental data for $|\mathbf{q}|<400$ $\mathrm{MeV}$ (including four points from the parametrization of Ref. [24]) to determine $\bar{d}_{9}$. We found, that 

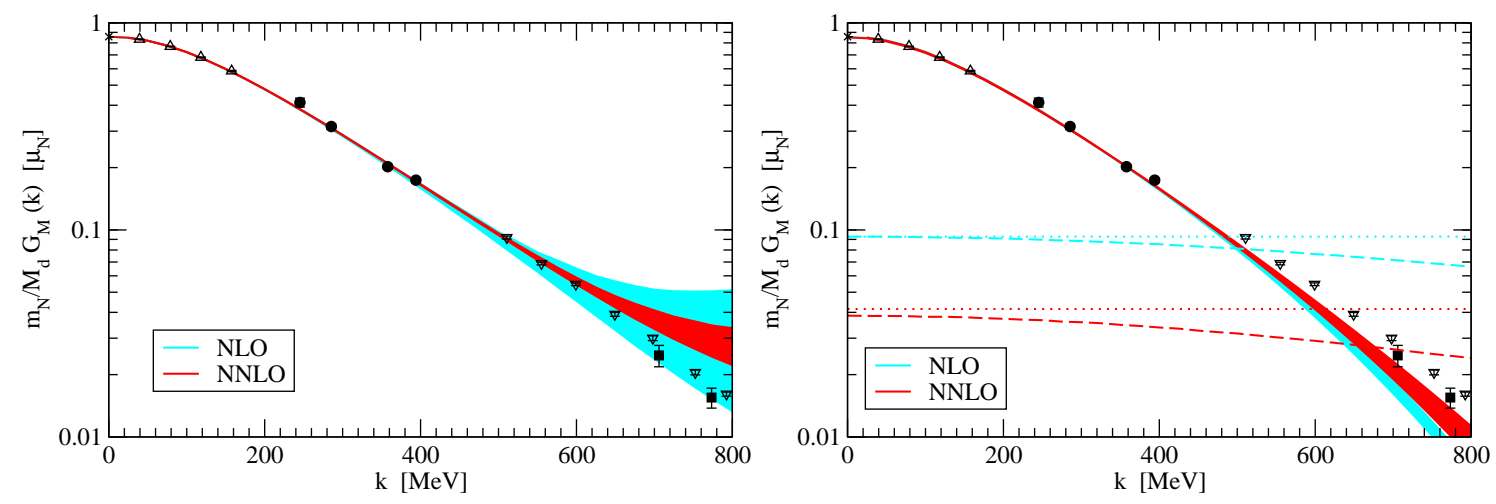

Figure 1: The magnetic form factor $G_{M}$ as a function of $|\mathbf{q}|$. On the left panel the result with $\mathbf{J}^{(s)}$ computed up to $O\left(e P^{4}\right)$ and on the right the impulse-approximation result. The dashed/dotted lines are the contributions from two-body pieces of $\mathbf{J}^{(s)}$, as described in the text. Experimental data for the magnetic moment is from [23]. The remaining data are from the parameterization of [24] (upward triangles) and scattering experiments reported in [25] (downward triangles), [26] (squares) and [27] (solid dots). The light blue (dark red) band represents the results with NLO (NNLO) wave functions.

using even lower- $|\mathbf{q}|$ data for this fit resulted in unstable answers, reflecting the insensitivity of $G_{M}$ to this LEC at small values of $|\mathbf{q}|$.

The results of this procedure are shown in the left panel of Fig. 1. The light/blue (dark/red) band is obtained using wave functions computed with the NLO (NNLO) $\chi$ EFT potential. The width of the band shows the variation of the prediction as $\Lambda$ and $\tilde{\Lambda}$ are changed in the range $\Lambda=400 \ldots 550$ $\mathrm{MeV}(\Lambda=450 \ldots 600 \mathrm{MeV})$ at NLO (NNLO) and $\tilde{\Lambda}=500 \ldots 700 \mathrm{MeV}$. The cutoff variation is reduced at NNLO, and the data well described for $Q^{2}<0.35 \mathrm{GeV}^{2}$.

In order to assess the momentum transfer at which the $\chi$ EFT expansion for $\mathbf{J}^{(s)}$ breaks down, in the right panel of Fig. 1 we show the size of different contributions to the final result. This time the bands represent the impulse-approximation result obtained with NLO (light/blue) and NNLO (dark/red) wave functions. The dotted (dashed) line is the effect from the piece of $\mathbf{J}_{2 B}^{(s)}$ that is proportional to $L_{2}\left(\bar{d}_{9}\right)$. For both two-body matrix elements, we show results averaged over the five cutoff combinations considered, with the light blue lines showing the NLO case, and the dark red lines obtained with NNLO wave functions. We estimate the breakdown scale of the EFT expansion by values of momentum transfer at which the $O\left(e P^{4}\right)$ two-body contributions start becoming comparable to the effect of the $O(e P)$ (impulse-approximation) piece of the current. The right panel of Fig. 1 shows that the smaller two-body contributions to $G_{M}$ found with the NNLO wave function delay the breakdown of the expansion. Even so, we would infer a breakdown scale $|\mathbf{q}|=600 \mathrm{MeV}$, as there the short-distance effect $\sim L_{2}$ becomes equal in magnitude to the impulseapproximation result.

In Table 1 we present the values of $\bar{d}_{9}$ and $L_{2}$ obtained in our fits. Small values of $\bar{d}_{9}$ are preferred, which is consistent with the findings of Ref. [28]. Reassuringly, the inferred values of $\bar{d}_{9}$ show only a very mild dependence on the cutoffs as compared to the expected natural size of this LEC, $\left|\bar{d}_{9}\right| \sim 1 \mathrm{GeV}^{-2}$. In contrast, the values of $L_{2}$ do depend on the choice of the regulator 
employed for the NN potential, as one would expect. It is comforting to see that all obtained values of $L_{2}$ are natural with respect to the cutoff scale $\Lambda$ employed in these calculations. The values of $L_{2}$ reported in the table show that two-body effects in $G_{M}$ play a larger role in the calculation with NLO deuteron wave functions, as seen in the right panel of Fig. 1.

\section{Summary}

The first two-body effects in the deuteron magnetic form factor $G_{M}$, occur at $O\left(e P^{4}\right)$ in $\chi \mathrm{EFT}$, i.e. three orders beyond leading. The description of $G_{M}$ is improved by the inclusion of these effects. Further, this allows for an exact reproduction of the deuteron magnetic moment. Experimental data is then well described for $Q^{2}<0.35 \mathrm{GeV}^{2}$, and the chiral expansion for $G_{M}$ is found to converge well for $Q^{2}<0.25 \mathrm{GeV}^{2}$, provided that the NNLO wave functions of Ref. [4] are employed.

\section{Acknowledgments}

S.K. would like to thank the organisers for a very enjoyable workshop and my collaborators Evgeny Epelbaum, Hermann Krebs, Ulf-G. Meißner and Daniel R. Phillips. This work is supported by the EU HadronPhysics3 project "Study of strongly interacting matter", by the European Research Council (ERC-2010-StG 259218 NuclearEFT), by the DFG (TR 16, "Subnuclear Structure of Matter").

\begin{tabular}{|c|c|c|c|}
\hline Order & $\Lambda / \tilde{\Lambda}[\mathrm{MeV}]$ & $\bar{d}_{9}\left[\mathrm{GeV}^{-2}\right]$ & $L_{2}\left[\mathrm{GeV}^{-4}\right]$ \\
\hline NLO & $400 / 500$ & -0.010 & 0.243 \\
NLO & $400 / 700$ & -0.011 & 0.249 \\
NLO & $550 / 500$ & 0.016 & 0.605 \\
NLO & $550 / 600$ & 0.017 & 0.731 \\
NLO & $550 / 700$ & 0.018 & 0.892 \\
NNLO & $450 / 500$ & -0.011 & 0.188 \\
NNLO & $450 / 700$ & -0.009 & 0.173 \\
NNLO & $550 / 600$ & 0.005 & 0.089 \\
NNLO & $600 / 500$ & 0.001 & 0.113 \\
NNLO & $600 / 700$ & -0.001 & 0.028 \\
\hline
\end{tabular}

Table 1: Values for $\bar{d}_{9}$ and $L_{2}$ found by fitting data up to $|\mathbf{q}|=400 \mathrm{MeV}$, using different values of the cutoffs. 


\section{References}

[1] E. Epelbaum, H.-W. Hammer and U.-G. Meißner, Rev. Mod. Phys. 81, 1773 (2009).

[2] E. Epelbaum and U.-G. Meißner, arXiv:1201.2136 [nucl-th].

[3] D. R. Entem and R. Machleidt, Phys. Rev. C 68, 041001 (2003).

[4] E. Epelbaum, W. Glöckle and U.-G. Meißner, Nucl. Phys. A 747, 362 (2005).

[5] T. -S. Park, D. -P. Min and M. Rho, Nucl. Phys. A 596, 515 (1996).

[6] S. Pastore, R. Schiavilla and J. L. Goity, Phys. Rev. C 78, 064002 (2008).

[7] S. Pastore, L. Girlanda, R. Schiavilla, M. Viviani and R. B. Wiringa, Phys. Rev. C 80, 034004 (2009).

[8] S. Pastore, L. Girlanda, R. Schiavilla and M. Viviani, Phys. Rev. C 84, 024001 (2011).

[9] M. Piarulli, L. Girlanda, L.E. Marcucci, S. Pastore, R. Schiavilla and M. Viviani, Phys. Rev. C 87, 014006 (2013).

[10] S. Kölling, E. Epelbaum, H. Krebs and U.-G. Meißner, Phys. Rev. C 80, 045502 (2009).

[11] S. Kölling, E. Epelbaum, H. Krebs and U.-G. Meißner, Phys. Rev. C 84, 054008 (2011).

[12] S. Kölling, E. Epelbaum and D.R. Phillips, Phys. Rev. C 86, 047001 (2012).

[13] D. R. Phillips, Phys. Lett. B 567, 12 (2003).

[14] D. R. Phillips, J. Phys. G G 34, 365 (2007).

[15] D. R. Phillips and T. D. Cohen, Nucl. Phys. A 668, 45 (2000).

[16] M. Walzl and U.-G. Meißner, Phys. Lett. B 513, 37 (2001).

[17] D. Abbott et al. [JLAB t20 Collaboration], Eur. Phys. J. A 7, 421 (2000). [18]

[18] Y. -H. Song, R. Lazauskas, T. -S. Park and D. -P. Min, Phys. Lett. B 656, 174 (2007).

[19] M. A. Belushkin, H. W. Hammer and U.-G. Meißner, Phys. Rev. C 75, 035202 (2007).

[20] R. A. Gilman and F. Gross, J. Phys. G 28, R37 (2002).

[21] M. Pavon. Valderrama, A. Nogga, E. Ruiz Arriola and D. R. Phillips, Eur. Phys. J. A 36, 315 (2008).

[22] S. Liebig, V. Baru, F. Ballout, C. Hanhart, A. Nogga [arXiv:1003.3826/nucl-th]

[23] I. Lindgren, in: K. Siegbahn (ed.), in: Alpha-, Beta- and Gamma-Ray Spectroscopy, Vol. 2, North-Holland, Amsterdam 1965.

[24] I. Sick, Prog. Part. Nucl. Phys. 47,(2001) 245.

[25] S.Auffret et. al, Phys. Rev. Lett 54,(1985) 649.

[26] R.Cramer et. al, Z. Phys. C 29,(1985) 513.

[27] G. G. Simon, C. Schmidt, V. H. Walther, Nucl. Phys. A 364,(1981) 285.

[28] A. Gasparyan and M. F. M. Lutz, Nucl. Phys. A 848, 126 (2010). 